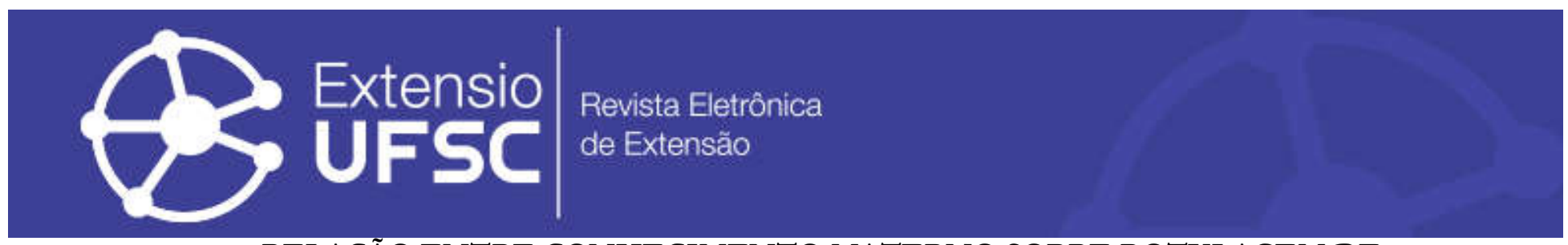

\title{
RELAÇÃO ENTRE CONHECIMENTO MATERNO SOBRE ROTULAGEM DE \\ ALIMENTOS E CONSUMO DE ULTRAPROCESSADOS EM CRIANÇAS E ADOLESCENTES ATENDIDOS EM UM PROJETO DE EXTENSÃO UNIVERSITÁRIA
}

\author{
Simone Barbara Jungblut \\ Universidade do Vale do Rio dos Sinos \\ simone_bj@hotmail.com
}

Paula Dal Bó Campagnolo

Universidade do Vale do Rio dos Sinos pcampagnolo@unisinos.br

\section{Resumo}

O objetivo desse estudo foi verificar a associação entre o conhecimento materno sobre rotulagem e o consumo de alimentos ultraprocessados em crianças e adolescentes participantes de um Programa de Ação Socioeducativa. A amostra foi realizada por conveniência, totalizando 21 participantes. Um questionário padronizado sobre rotulagem foi utilizado com as mães e foram aplicados 2 recordatórios alimentares de $24 \mathrm{~h}$ com as crianças/adolescentes. O consumo de alimentos ultraprocessados em gramas, kcal e percentual de kcal foi quantificado em relação ao consumo energético total. Verificouse que a mediana de consumo de kcal provenientes de alimentos ultraprocessados e gramas desses alimentos foram maiores entre os filhos de mães que disseram não ter o hábito de ler os rótulos dos alimentos. Pode-se concluir que ações educativas que promovam maior conhecimento e interpretação de rótulos são necessárias para melhores escolhas alimentares.

Palavras-chave: Rotulagem de Alimentos. Alimentos Ultraprocessados. Programa de Ação Socioeducativo.

\section{RELATIONSHIP BETWEEN MATERNAL KNOWLEDGE ON FOOD LABELING AND ULTRAPROCESSED FOOD INTAKE IN CHILDREN AND ADOLESCENTS}

\section{Abstract}

The aim of this study was to verify the association between maternal knowledge about labeling and the consumption of ultra-processed foods by children and adolescents of a Socio-Educational Action Program. The sample was conducted for convenience, totalizing 21 participants. A standardized labeling questionnaire was used with mothers and two " $24 \mathrm{~h}$ food recalls" were applied with children/adolescents. The consumption of ultra-processed foods in grams, kcal and percentage of kcal was quantified in relation to the total energy consumption. The median consumption of kcal from ultra-processed foods and grams of these foods was higher among children of mothers who said they had no habit of reading food labels. It can be concluded that educational actions that promote greater knowledge and interpretation of labels are necessary for better food choices.

Keywords: Food Labeling. Ultra-Processed Food. Socio-Educational Action Program.

\section{RELACIÓN ENTRE CONOCIMIENTO MATERNO SOBRE ETIQUETADO DE ALIMENTOS Y CONSUMO DE ULTRAPROCESADO EN NIÑOS Y ADOLESCENTES}

\section{Resumen}

El objetivo de este estudio fue verificar la asociación entre el conocimiento materno sobre el etiquetado y el consumo de alimentos ultraprocesados por niños y adolescentes que participan de un Programa de Acción Socioeducativa. La muestra se realizó por conveniencia, totalizando 21 participantes. Se utilizó un cuestionario de etiquetado estandarizado con madres y se aplicaron 2 "retiradas las 24 horas" con niños/adolescentes. El consumo de alimentos ultraprocesados en gramos, kcal y porcentaje de kcal se cuantificó en relación con el consumo total de energía. El consumo medio de kcal de alimentos ultraprocesados y gramos de estos alimentos fue mayor entre los hijos de madres que dijeron que no tenían la costumbre de leer las etiquetas de los alimentos. Se puede concluir que las acciones educativas que promueven un mayor conocimiento e interpretación de las etiquetas son necesarias para una mejor elección de alimentos.

Palabras clave: Etiquetado de Alimentos. Alimentos Ultraprocesados. Programa de Acción Socioeducativa. 
Relação entre conhecimento materno sobre rotulagem de alimentos e consumo de ultraprocessados em crianças e adolescentes atendidos em um projetos de extensão universitária

\section{INTRODUÇÃO}

Dados do estudo Trends in adult body-mass index in 200 countries from 1975 to 2014: a pooled analysis of 1698 population-based measurement studies with $19 \cdot 2$ million participants, de 2016, alertam que, se a tendência atual de obesos continuar, em 2025 a prevalência de obesidade no mundo atingirá $18 \%$ em homens e extrapolará 21\% nas mulheres e a obesidade severa ultrapassará 9\% nos homens e 6\% nas mulheres(NCD RISK FACTOR COLLABORATION, 2016).O aumento nas taxas de excesso de peso e doenças crônicas não transmissíveis (DCNT) é oriundo, entre outros fatores, da inversão dos padrões alimentares, representada principalmente pela substituição cada vez maior da alimentação tradicional por produtos alimentícios altamente processados e prontos para o consumo (SPARRENBERGER et al., 2015).

No Brasil, o consumo de alimentos ultraprocessados é crescente. Conforme o Guia Alimentar para População Brasileira (BRASIL, 2014), esses alimentos possuem composição nutricional desbalanceada inerente à natureza dos ingredientes, e por esse motivo favorecem cardiopatias, diabetes e alguns tipos de câncer. Esses alimentos também favorecem o consumo excessivo de calorias e, por serem consumidos em grande quantidade por crianças e adolescentes, merecem atenção e cautela (BRASIL, 2014).

Os alimentos ultraprocessados também, em sua maioria, são ricos em sódio, e a ingestão de sódio em excesso está associada ao desenvolvimento de hipertensão arterial. Problemas hipertensivos na vida adulta estão relacionados a desordens de pressão arterialna infância, iniciando o quadro a partir da alimentação inadequada (PINTO, et al., 2011; SPARRENBERGER et al., 2015). O consumo de ultraprocessados também tem sido associado a alterações no perfil lipídico de crianças (RAUBER et al., 2014), à síndrome metabólica em adolescentes (TAVARES et al., 2012) e às altas taxas de obesidade em adolescentes e adultos (LOUZADA et al., 2015).

Em um ambiente familiar, a disponibilidade domiciliar de alimentos é resultado de um conjunto de fatores que determinam as escolhas alimentares por parte dos pais, uma vez que são eles que definem quais alimentos estarão disponíveis para as crianças. Sendo assim, variáveis como escolaridade materna e conhecimento sobre alimentação saudável podem influenciar a escolha de alimentos e consequentemente a qualidade da alimentação das crianças (CAMPBELL et al., 2013). Os fatores que determinam as escolhas alimentares são diversos: componentes da dieta (como sabor), pressões culturais e sociais, fatores cognitivo-afetivos einfluências familiares, genéticas e epigenéticas. As escolhas alimentares também são influenciadas pela forma como os alimentos são comercializados e rotulados e por fatores econômicos. Além disso, mecanismos 
Relação entre conhecimento materno sobre rotulagem de alimentos e consumo de ultraprocessados em crianças e adolescentes atendidos em um projetos de extensão universitária

fisiológicos, incluindo sinalizações que partem do trato gastrointestinal e do tecido adiposo, que afetam não apenas a sensação de fome e saciedade, mas tambéma motivação para comer nutrientes específicos também são fatores que determinam as escolhas alimentares (LENG et al., 2017).

A rotulagem do alimento é a primeira forma de comunicação entre o consumidor e a indústria no que tange à aquisição de alimento no ato da compra. A propaganda também colabora nessa comunicação, podendo contribuir com o consumo de alimentos saudáveis ou estimular o consumo de uma dieta pobre em qualidade, variedade e que compromete a saúde do indivíduo. (BENDINO; POPOLIM; OLIVEIRA, 2012). Os rótulos presentes nos alimentos industrializados precisam ser claros e facilmente compreensíveis, pois na ausência de compreensão, o consumidor acaba não atribuindo importância ao que nele contém - o que prejudica o hábito de uma alimentação saudável e a prevenção de agravos à saúde.

A fim de buscar as melhores estratégias de prevenção e promoção da saúde, é fundamental conhecer a magnitude do consumo de alimentos ultraprocessados, bem como seus determinantes. Sendo assim, o presente estudo tem como objetivo avaliar o nível de conhecimento materno sobre rotulagem de alimentos e verificar associação entre o conhecimento materno sobre rotulagem nutricional e o consumo de alimentos ultraprocessados em crianças e adolescentes.

\section{MATERIAIS E MÉTODOS}

Trata-se de um estudo observacional transversal, com abordagem quantitativa. Os dados foram coletados por meio de entrevistas realizadas com as mães dos participantes de um Programa de Ação Socioeducativa que ocorre dentro de uma escola municipal em São Leopoldo/RS.

A amostragem foi por conveniência e a população de estudo foi composta por pares de mães e crianças/adolescentes (entre 7 e 14 anos) participantes do Programa, oferecido por uma Universidade Comunitária, em parceria com a Secretária Municipal de Educação da cidade de São Leopoldo. Esse Programa atua a partir das demandas de crianças e adolescentes em situação de vulnerabilidade social e ocorre em conformidade com a Política Nacional de Assistência Social, nas ações de proteção social (BRASIL, 2009).

O Programa utiliza uma horta chamada Horta Mãe-da-Terra como instrumento pedagógico principal, e promovem atividades como o cultivo/produção de verduras, legumes, chás, temperos, compostagem, conhecimento e conscientização sobre a preservação e o uso racional 
Relação entre conhecimento materno sobre rotulagem de alimentos e consumo de ultraprocessados em crianças e adolescentes atendidos em um projetos de extensão universitária

dos recursos naturais, manutenção de áreas de nascentes, oficinas de cidadania, mutirões ecológicos, entre outras. Os profissionais que formam a equipe são dois biólogos, um psicólogo, uma assistente social.

Após a aprovação do Projeto de Pesquisa pelo Comitê de Ética em Pesquisa no mês de agosto de 2019, foram agendadas as coletas de dados com as mães e participantes do Programa. Três mães solicitaram que fossem visitadas em casa por dificuldade de ir até a Escola e as demais mães compareceram ao encontro marcado na escola com a estudante de Nutrição. A Assistente Social do Programa foi quem levou a graduanda nos domićlios para aplicar os questionários com as mães, permanecendo presente durante todo o período que se fez necessário no local da entrevista. A coleta de dados iniciou em agosto e foi finalizada em setembro de 2019.

Foram aplicados um questionário para avaliar o conhecimento materno sobre rotulagem de alimentos (com as mães) e o recordatório alimentar de $24 \mathrm{~h}$ - R24h (com as crianças/adolescentes) para avaliação do consumo de alimentos ultraprocessados no primeiro encontro e, no segundo encontro, foi realizado outro R24h. O tempo de duração total do primeiro encontro foi de 50 minutos e do segundo encontro, 25 a 30 minutos. Houve o intervalo de no mínimo sete dias entre a aplicação do primeiro e do segundo R24h. Para o R24h, foi utilizada a técnica de passagens múltiplas, que consiste em estimular o entrevistado a recordar os alimentos ingeridos no dia anterior em três fases distintas: listagem rápida, descrição detalhada e revisão (KAC, SICHIERI, GIGANTE, 2007). Dependendo da idade e condição da criança, a mãe poderia auxiliar nas respostas quando achasse necessário.

O cálculo do consumo alimentar foi realizado de acordo com a natureza, extensão e a finalidade do processamento (Monteiro et al., 2012).Para os fins deste estudo, apenas o consumo de ultraprocessados foi analisado, caracterizados como produtos alimentares formulados principalmente ou inteiramente a partir de ingredientes processados, tipicamente incluindo pouco ou nenhum alimento completo (por exemplo, pão, batatas fritas, biscoitos, doces, chocolate, refrigerantes, cereais matinais e carne processada). São exemplos de alimentos ultraprocessados: biscoitos doces e salgados, salgadinhos tipo chips, barras de cereal, guloseimas em geral, lanches tipo fast food, macarrão instantâneo, vários tipos de pratos prontos ou semiprontos e refrigerantes. A quantidade em gramas de cada alimento e o valor energético total foram calculados e a média dos dois inquéritos recordatórios de 24 horas foi utilizada nas análises.

Para calcular as medidas caseiras foi utilizado o livro físico Tabela para avaliação de Consumo Alimentar em Medidas Caseiras (PINHEIRO et al., 2005) e os rótulos dos alimentos que não constavam na Tabela foram consultados diretamente por meio do site ou solicitados que os participantes trouxessem no próximo encontro - perguntava-se a marca e demais detalhes de 
Relação entre conhecimento materno sobre rotulagem de alimentos e consumo de ultraprocessados em crianças e adolescentes atendidos em um projetos de extensão universitária

cada item coletado no R24h. Para a composição dos alimentos foram utilizadas as Tabelas TACO - Tabela Brasileira de Composição dos Alimentos (UNICAMP, 2011) (prioritariamente) e a Tabela de composição de alimentos - Suporte para decisão nutricional (PHILIPPI, 2018). A partir dos R24h foi quantificado o consumo de alimentos ultraprocessados em gramas, kcal e percentual de kcal em relação ao consumo energético total.

O conhecimento materno sobre rotulagem foi avaliado por questionário padronizado e aplicado no encontro com a mãe. O questionário foi composto por 16 questões relativas a conhecimento de rotulagem e 3 questões relativas a hábitos de compra de alimentos e origem dos alimentos. Os artigos utilizados para embasar a construção do questionário de conhecimento de rotulagem foram: Avaliação do conbecimento e dificuldades de consumidores frequentadores de supermercado convencional em relação à rotulagem de alimentos e informação nutricional (BENDINO; POPOLIM; OLIVEIRA, 2012) e o artigo Conbecimento de consumidores idosos sobre rotulagem de alimentos (NASCIMENTO et al., 2013).

As seguintes variáveis foram coletadas com a finalidade de caracterizar a população estudada: sexo da criança (informado pela entrevistada em masculino ou feminino), idade da criança (informada pela entrevistada em anos completos), cor de pele da criança (informada pela entrevistada como branca, parda, preta ou outras), idade da mãe (informada pela entrevistada em anos completos), escolaridade da mãe (informada pela entrevistada em anos de estudo),se a mãe sabe ler, ocupação materna (categorizada em trabalha fora, desempregada, pensionista, dona de casa e outros), renda mensal da família (informada pela entrevistada em reais), ser beneficiado do Bolsa Família (informada pela entrevistada).

O banco de dados foi realizado no EpiData versão 3.1, e as análises foram realizadas no software Statistical Package for Social Scienc (SPSS) e, versão 22.0. As variáveis numéricas foram descritas por média e desvio-padrão ou mediana e intervalo interquartil, e as variáveis categóricas por meio de frequências absolutas e relativas. O teste Mann-Whitney foi realizado para verificar a associação entre consumo de ultraprocessados e nível de conhecimento materno. Foram consideradas estatisticamente significantes associações com nível de significância menor de 5\%.

Por se tratar de pesquisa envolvendo seres humanos, esse estudo seguiu as determinações da Resolução 466/2012 do Conselho Nacional de Saúde, que legisla sobre diretrizes e normas regulamentadoras de pesquisa envolvendo humanos (CNC, 2012). O presente estudo foi aprovado pelo Comitê de Ética em Pesquisa da Universidade do Vale do Rio dos Sinos sob o parecer de $n^{\circ}$ 3.516.425. Antes do início da entrevista com cada uma das mães era lido o Termo de Consentimento Livre e Esclarecido (TCLE) e quando a mãe sanava todas as suas dúvidas, ela era encaminhada a assinar o Termo. As mães assinavam o TCLE, bem como as crianças entre 7 e 
Relação entre conhecimento materno sobre rotulagem de alimentos e consumo de ultraprocessados em crianças e adolescentes atendidos em um projetos de extensão universitária

10 anos assinaram o termo de assentimento e adolescentes entre 11 e 14 anos assinaram o termo de assentimento respectivo para sua faixa etária - todos ficaram com uma cópia do documento assinado e uma cópia ficava com a entrevistadora.

\section{RESULTADOS E DISCUSSÃO}

Participaram do estudo 21 integrantes do Projeto Socioeducativo com idade média de 10 anos (DP 1,6), sendo 71,4\% do sexo masculino e 47,6\% de cor de pele não branca. A média de idade das mães foi de 39,1 anos (DP 12,3), 90,5\% relataram saber ler, 42,9\% referiram estar em união estável, 42,9\% eram dona de casa e 52\% disseram ser responsáveis pelas compras dos alimentos (ida ao supermercado e escolha dos itens). O mercado do bairro é o local onde ocorre a compra dos alimentos para 90,5\% das mães, enquanto que 9,5\% responderam que vão até um mercado longe de casa. A maior parte dos alimentos das famílias são comprados, o que representa $81 \%$; e $19 \%$ das famílias tem a maior parte dos alimentos das suas casas provenientes de doações recebidas.A renda média mensal das famílias foi de $\mathrm{R} \$ 1.113,80$ (DP 428,4); e 9 famílias (42,9\%) têm o acréscimo na disponibilidade financeira mensal de recursos do Bolsa Família(Tabela 1).

Tabela 1. Características da amostra

\begin{tabular}{lcc}
\hline Crianças e adolescentes $(\mathbf{n}=\mathbf{2 1})$ & & \\
Idade; média (DP) & 10,57 & $(1,6)$ \\
Cor da pele; não branca, n (\%) & 10 & $(46,6)$ \\
Sexo; feminino, n (\%) & 6 & $(28,6)$ \\
Variáveis maternas $(\mathbf{n}=\mathbf{2 1})$ & & \\
Idade; média (DP) & 39,1 & $(12,3)$ \\
Estado civil, n (\%) & & \\
Solteira & 1 & $(4,8)$ \\
Casada & 6 & $(28,6)$ \\
União estável & 9 & $(42,9)$ \\
Divorciada & 2 & $(9,5)$ \\
Viúva & 3 & $(14,3)$ \\
Sabe ler, n (\%) & 19 & $(90,5)$ \\
Escolaridade materna; $<8$ anos, n(\%) & 13 & $(42,9)$ \\
Ocupação, n $(\%)$ & & \\
Trabalha fora de casa & 2 & $(9,5)$ \\
Desempregada & 3 & $(14,3)$ \\
Pensionista & 2 & $(9,5)$ \\
Dona de casa & 9 & $(42,9)$ \\
Outro & 5 & $(23,8)$ \\
Renda mensal da família; R\$, média (DP) & 1113,8 & $(428,4)$ \\
& &
\end{tabular}


Relação entre conhecimento materno sobre rotulagem de alimentos e consumo de ultraprocessados em crianças e adolescentes atendidos em um projetos de extensão universitária

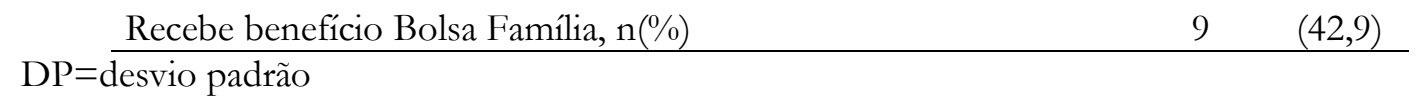

Dezenove mães responderam o questionário sobre conhecimento materno de rotulagem dos alimentos e sobre o Guia Alimentar para População Brasileira. Na tabela 2, observa-se que 89,5\% das mães responderam saber "o que é ingrediente culinário", o que pode se justificar pelo fato de que $42,9 \%$ da amostra materna ser composta de donas de casa e, portanto, teriam maior familiaridade com a preparação de refeições, o que também colabora para que 78,9\% respondentes tenham dito saber interpretar uma lista de ingredientes. O Guia Alimentar para a População Brasileira(BRASIL, 2014) enfatiza a importância de limitar o uso de determinados alimentos, como os processados, incentivando o seu consumo em pequenas quantidades, como ingredientes de preparações culinárias ou como parte de refeições baseadas em alimentos in natura ou minimamente processados. Para tanto, faz-se essencial saber interpretar e entender as informações contidas nos rótulos, tendo em vista que quanto maior o repertório de conhecimento ligado à alimentação, maior é a chance de que as escolhas alimentares sejam pautadas em saúde e não somente em influências midiáticas, entre outras. Por outro lado, vale ressaltar, que além do conhecimento sobre rotulagem, questões econômicas, sociais, ambientais, também influenciam as escolhas (AGÊNCIA NACIONAL DE VIGILÂNCIA SANITÁRIA (ANVISA), 2005; BRASIL, 2012; BRASIL, 2014).

Tabela 2. Conhecimento dos termos utilizados no Guia Alimentar para a População Brasileira.

\begin{tabular}{lcc}
\hline & $\mathrm{n}$ & $\%$ \\
\hline Sabe o que é alimento in natura? & 10 & 52,6 \\
Sabe o que é alimento minimamente processado? & 3 & 15,8 \\
Sabe o que é alimento processado? & 10 & 52,6 \\
Sabe o que é ingrediente culinário? & 17 & 89,5 \\
Sabe o que é alimento "ultraprocessado"? & 10 & 52,6 \\
\hline
\end{tabular}

Apenas 31,6\% das mães costumam ler os rótulos dos alimentos, 10,5\% confiam nas informações dos rótulos quando lêem, 52,6\% entende parcialmente as informações ao ler, 10,5\% responderam entender tudo e 36,8\% citam não entender (Tabela 3).O rótulo é a forma de comunicação entre o consumidor e o produto, sendo que nos dias atuais, em que é grande o cuidado com a saúde, a informação clara e verdadeira transforma-se em uma aliada da mesma. É a partir de uma leitura correta e íntegra do rótulo que o consumidor fica sabendo se naquele alimento há algum ingrediente que seja nocivo. Um estudo realizado na cidade de Viçosa, Minas 
Relação entre conhecimento materno sobre rotulagem de alimentos e consumo de ultraprocessados em crianças e adolescentes atendidos em um projetos de extensão universitária

Gerais, com 328 crianças, observou-se que quando a escolaridade das mães atingia 12 ou mais anos de estudos, a chance da alimentação dos filhos conter alimentos ultraprocessados aumentou quatro vezes quando comparada com as mães com menor escolaridade. Filhos de mães com menor escolaridade apresentaram consumo alimentar mais próximo do considerado adequado (VILLA et al., 2015).

Tabela 3. Conhecimento materno sobre rotulagem dos alimentos.

\begin{tabular}{lcc}
\hline Mães (n = 19) & $\mathbf{n}$ & $\mathbf{\%}$ \\
\hline Costuma ler rótulos dos alimentos? & 6 & 31,6 \\
Confia nas informações dos rótulos? & 2 & 10,5 \\
Entende as informações quando lê: & 7 & 36,8 \\
$\quad$ Não entende & 2 & 10,5 \\
$\quad$ Sim, entende tudo & 10 & 52,6 \\
Entende parcialmente & 10 & 52,6 \\
Sabe o que é valor energético? & 5 & 23,8 \\
Identifica valor energético no rótulo? & 15 & 78,9 \\
Sabe o que é lista de ingredientes? & 11 & 64,7 \\
Sabe interpretar lista de ingredientes? & 1 & 5,3 \\
Sabe o que é alegação de saúde? & & \\
\hline Mães (n = 19) & $\mathbf{n}$ & $\mathbf{0}$ \\
\hline & & \\
Elementos de rotulagem que acha importante verificar no rótulo para & & \\
manutenção de uma alimentação saudável: & & \\
$\quad$ Porção & 12 & 63,2 \\
Carboidrato & 17 & 89,5 \\
Proteína & 16 & 84,2 \\
Gordura & 10 & 52,6 \\
Gordura trans & 11 & 57,9 \\
Fibra & 10 & 52,6 \\
Sódio & 13 & 68,4 \\
Vitaminas & 15 & 78,9 \\
Valor calórico & 13 & 68,4 \\
$\quad$ Lista de ingredientes & 12 & 63,2 \\
\hline
\end{tabular}

A respeito das informações que as mães entrevistadas utilizam como critério para não comprar determinado alimento, observou-se que as mais frequentes citadas foram a validade $(47,4 \%)$,a presença de dois rótulos na mesma embalagem- quando um é colado sobre o original $(15,8 \%)$ e o preço elevado (10,5\%). Também, 10,5\% das mães responderam que não deixam de comprar o alimento por informações que estão contidas no rótulo (Tabela 3). No estudo realizado por Marzarotto \& Alves (2017) com frequentadores de um estabelecimento 
Relação entre conhecimento materno sobre rotulagem de alimentos e consumo de ultraprocessados em crianças e adolescentes atendidos em um projetos de extensão universitária

local, foi observado que o valor energético $(27,8 \%)$, seguido do sódio $(15 \%)$ e gorduras $(14,8 \%)$ foram as informações mais lidas, porém, também identificaram uma parte dos entrevistados que não costuma ler os rótulos $(19,4 \%)$. Semelhante aos achados do presente estudo, Nascimento et al. (2013) constataram que o prazo de validade é consultado por quase $90 \%$ dos consumidores participantes, sendo utilizado como critério de compra.

Muitas mães, como já citado, compram alimentos em um mercado próximo a sua residência, hábito que colabora para que conheçam a dinâmica do estabelecimento e tenham confiança nos procedimentos adotados pelo mesmo. 15,8\% das mães citaram que não compram alimentos com dois rótulos, indicando que não se sentem seguras e que podem estar colocando a saúde dos seus familiares em risco com um alimento fraudado.

Tabela 4. Informações do rótulo que fazem com que a mãe não compre determinado alimento

\begin{tabular}{lcc}
\hline Itens citados pelas mães $(\mathbf{n}=\mathbf{1 9})$ & $\mathbf{N}$ & $\mathbf{0}$ \\
\hline Validade & 9 & 47,4 \\
Se contém leite & 1 & 5,3 \\
Preço elevado & 2 & 10,5 \\
Se tem muito sódio e gordura Trans & 1 & 5,3 \\
Quando tem 2 rótulos na mesma embalagem & 3 & 15,8 \\
Contém pimenta & 1 & 5,3 \\
Não deixa de comprar alimentos por informações & & \\
que contém no rótulo & 2 & 10,5 \\
\hline
\end{tabular}

A mediana de consumo de ultraprocessados foi de 663,2kcal (II=340,7), 416,5g $(\mathrm{II}=510,8)$ e em termos de percentual em relação ao consumo energético total da dieta, a mediana de consumo foi de 40,9\% ( $\mathrm{II}=20,1)$. Foi observado que a mediana de consumo de kcal provenientes de alimentos ultraprocessados e gramas desses alimentos foi maior entre os filhos de mães que disseram não ter o hábito de ler os rótulos dos alimentos. Também, foi observado que o consumo de kcal de ultraprocessados foi superior entre os filhos de mães que disseram não saber o que é um alimento ultraprocessado (Tabela 5).

Resultados semelhantes foram encontrados no estudo de Miller et al. (2015b), que foi um dos primeiros a demonstrar que o uso dos rótulos dos alimentos está relacionado à qualidade da dieta. Foi observado que aqueles que prestavam mais atenção aos rótulos dos alimentos tinham maior probabilidade de consumir uma dieta saudável. Em uma revisão da literatura, Miller et al. (2015a) concluiram que o conhecimento nutricional que o indivíduo possui fornece suportepara que ele utilize os rótulos de alimentos e impacte positivamente na compra de alimentos. No entanto, as medidas de conhecimento utilizadas pelos estudos que compuseram a 
Relação entre conhecimento materno sobre rotulagem de alimentos e consumo de ultraprocessados em crianças e adolescentes atendidos em um projetos de extensão universitária

revisão foram variadas, o que dificulta identificar quais aspectos nutricionais são mais importantes de serem abordados. De qualquer forma, esses dados levantam a hipótese de que a melhora do conhecimento nutricionalpela população pode impactar na maior utilização dos rótulos de alimentos e consequentemente na qualidade da dieta.

Não somente a leitura dos rótulos dos alimentos no momento da compra, mas também o envolvimento das crianças nessa tarefa pode ser importante. Estudo com universitários indicou que aqueles que liam regularmente os rótulos dos alimentos no momento da compra, apresentaram melhor qualidade da dieta quando comparado aos que nunca liam os rótulos (BUYUKTUNCER et al., 2018). Assim, quanto mais precocemente este hábito for incorporado à rotina das crianças, os efeitos tendem a ser melhores.

No estudo de Russell et al. (2015), saúde, nutrição e sabor foram os principais motivadores para os pais escolherem os alimentos que são adquiridos, enquanto preço, e publicidade estavam entre os motivos considerados menos importantes. Quanto mais as escolhas alimentares dos pais para os filhos eram motivadas pelo que seus filhos desejavam, menos os filhos gostavam de vegetais, frutas e cereais. Por outro lado, quando os motivos de compra estavam relacionados à saúde e nutrição,se identificou maior preferência por parte das crianças por frutas e vegetais. Embora os pais pareçam bem-intencionados em seus motivos para selecionar alimentos para as crianças, existem lacunas a serem abordadas, especialmente o fato de se comprar alimentos somente de acordo com os desejos de seus filhos. Os resultados desse estudo corroboram com a ideia de que programas de educação alimentar com os responsáveis são essenciais para impactar na qualidade da dieta das crianças.

Os pais desempenham o papel de promotores de saúde, além de servirem como exemplo para seus filhos, influenciando suas escolhas alimentares. Resultados de uma metanálise indicaram que a disponibilidade de alimentos e o exemplo dos pais são as variáveis mais fortemente associadas ao consumo saudável e não saudável de alimentos por parte das crianças (YEE et al., 2017). Estudo qualitativo que investigou influências nas dietas de crianças pequenas em famílias de baixa renda identificou que alguns pais não entendem o que constitui uma dieta saudável. No entanto, a maioria dos pais incluía frutas e vegetais em graus variados e estava motivada a fornecer alimentos saudáveis aos filhos, sugerindo que, com apoio e informações adequados, as dietas dessas crianças poderiam ser melhoradas (LOVELACE\& RABIEE-KHAN, 2015).

Por mais que o tamanho da amostra do presente estudo tenha sido pequeno, a relação encontrada entre o conhecimento materno sobre rotulagem de alimentos e o consumo de ultraprocessados pelas crianças e adolescentes se mostra relevante, uma vez que diferentes 
Relação entre conhecimento materno sobre rotulagem de alimentos e consumo de ultraprocessados em crianças e adolescentes atendidos em um projetos de extensão universitária

estudos têm demonstrado associação entre o consumo de ultraprocessados e baixa qualidade da dieta, obesidade edislipidemia (RAUBER et al., 2014). Considerando que, no Brasil, estamos em pleno momento de discussão a respeito de uma nova proposta de rotulagem de alimentos, não podemos deixar passar essa oportunidade de modificar a rotulagem com o intuito de torná-la mais acessível a diferentes públicos, visto que parecem impactar diretamente no consumo alimentar das crianças.

Por se tratar de um estudo transversal, não foi possível avaliar a causa e efeito em relação ao conhecimento de rotulagem por parte das mães e a ingestão de ultraprocessados. Outro viés desse estudo é o de memória, visto que depende da memória da criança/adolescente, e mesmo da mãe, no momento do R24h. De qualquer forma, o recordatório alimentar de $24 \mathrm{~h}$ é um instrumento muito utilizado e validado no meio científico, desde que realizado ao menos em dois momentos para reduzir o viés relacionado à variabilidade intraindividual do consumo alimentar. Cabe também ressaltar que se trata de uma amostra pequena, com amostragem por conveniência, de um local específico. Sendo assim, os resultados não podem ser extrapolados para outros contextos, mas, sim, servem para gerar hipótesese servem como base para novos estudos com populações maiores.

\section{CONSIDERAÇÕES FINAIS}

Os alimentos industrializados e, principalmente, os classificados como ultraprocessados, possuem ingredientes que podem afetar a saúde de maneira negativa. A Resolução da Diretoria Colegiada (RDC) da Agência Nacional de Vigilância Sanitária (ANVISA) n. 259, de 20 de setembro de 2002, regulamenta a rotulagem de alimentos, tornando obrigatório uma série de regras afim de assegurar a saúde do consumidor, inclusive deliberando que no rótulo deva ser encontrado todos os ingredientes que o alimento contém (BRASIL, 2012). Alertas sobre alto teor de sódio, gordura e açúcar, conforme vem sendo proposto pela ANVISA facilitaria e identificação de alimentos ultraprocessados e apoiaria o processo de seleção e escolha de alimentos, podendo prevenir o consumo excessivo desses alimentos.

A partir dos resultados encontrados no presente estudo, ações de educação em saúde relacionadas a interpretação de rótulos de alimentos e dos conceitos que embasam o Guia Alimentar para População Brasileira tornam-se necessárias e urgentes para a amostra estudada, podendo ser estendido para a população em geral, pois o conhecimento das mães sobre esses aspectos parece estar refletindo no consumo alimentar das crianças. Mesmo todas as mães da 
Relação entre conhecimento materno sobre rotulagem de alimentos e consumo de ultraprocessados em crianças e adolescentes atendidos em um projetos de extensão universitária

amostra sendo de baixo nível socioeconômico, algumas possuíam maior conhecimento acerca do tema, o que demonstra existir a possibilidade de aprender e aplicar esses conhecimentos. 
Relação entre conhecimento materno sobre rotulagem de alimentos e consumo de ultraprocessados em crianças e adolescentes atendidos em um projetos de extensão universitária

Tabela 5. Consumo de ultraprocessados por crianças e adolescentes de acordo com o conhecimento materno

\begin{tabular}{|c|c|c|c|c|c|c|c|c|c|}
\hline & \multicolumn{3}{|c|}{ \% do VET de ultraprocessados } & \multicolumn{3}{|c|}{ gramas de ultraprocessados } & \multicolumn{3}{|c|}{ kcal de ultraprocessados } \\
\hline & mediana & II & $\mathrm{P}$ & Mediana & II & $\mathrm{P}$ & mediana & II & $\mathrm{p}$ \\
\hline \multicolumn{10}{|c|}{ Costuma ler o rótulo dos alimentos? } \\
\hline Sim & 40,7 & 33,2 & 0,282 & 330,5 & 458,7 & 0,003 & 493,2 & 275,8 & $<0,001$ \\
\hline Não & 46,4 & 17 & & 775,7 & 322,7 & & 1195,5 & 889,9 & \\
\hline \multicolumn{10}{|c|}{ Entende as informações do rótulo? } \\
\hline Sim/parcialmente & 42,2 & 16,9 & 0,902 & 555,6 & 435,8 & 0,068 & 810,4 & 799,6 & 0,068 \\
\hline Não & 40,9 & 40,6 & & 253,7 & 571 & & 493,2 & 561,5 & \\
\hline \multicolumn{10}{|c|}{ Sabe o que são alimentos in natura? } \\
\hline Sim & 37,9 & 34,3 & 0,720 & 344,9 & 460,9 & 0,079 & 512,2 & 323,4 & 0,053 \\
\hline Não & 43,6 & 12,9 & & 585,5 & 467,3 & & 924,2 & 1083,8 & \\
\hline \multicolumn{10}{|c|}{ Sabe o que são alimentos ultraprocessados? } \\
\hline $\operatorname{Sim}$ & 33,9 & 25,7 & 0,133 & 358,1 & 455,0 & 0,095 & 512,2 & 323,4 & 0,053 \\
\hline Não & 46,7 & 13,5 & & 640,0 & 495,8 & & 924,2 & 1065,8 & \\
\hline
\end{tabular}


Relação entre conhecimento materno sobre rotulagem de alimentos e consumo de ultraprocessados em crianças e adolescentes atendidos em um projetos de extensão universitária

\section{REFERÊNCIAS}

AGÊNCIA NACIONAL DE VIGILÂNCIA SANITÁRIA (ANVISA). Manual de orientação aos consumidores: educação para o consumo saudável. Brasília (DF): Ministério da Saúde; Universidade de Brasília, 2005. Disponível em: http://portal.anvisa.gov.br/ documents/33916/396679/manual consumidor.pdf/e31144d3-0207-4a37-9b3b-e4638d48934b.

Acesso: 30 out. 2019.

BENDINO, N. I.; POPOLIM, W. D.; OLIVEIRA, C. R. Á. Avaliação do conhecimento e dificuldades de consumidores frequentadores de supermercado convencional em relação à rotulagem de alimentos e informação nutricional. J Health Sci Inst, v. 30, n. 3, p. 261-5, 2012. Disponível em: https://www3.unip.br/presencial/comunicacao/publicacoes/ics/ edicoes/2012/03 jul-set/V30 n3 2012 p261a265.pdf. Acesso em: 20 maio 2019.

BRASIL. Agência Nacional de Vigilância Sanitária. Resolução-RDC $\mathbf{n}^{\mathbf{0}} 259$ de 20 de setembro de 2002.Diário Oficial da União, Brasília, DF, 23 set. 2012. Seção 1, p. 33. Disponível em: http://portal.anvisa.gov.br/documents/33880/2568070/RDC 259 2002.pdf/e40c2ecb-6be64a3d-83ad-f3cf7c332ae2. Acesso em: 05 nov. 2019.

BRASIL. Ministério da Saúde. Secretaria de Atenção à Saúde. Departamento de Atenção Básica. Guia alimentar para a população brasileira. 2. ed. Brasília, DF, 2014. Disponível em: http://bvsms.saude.gov.br/bvs/publicacoes/guia_alimentar_populacao_brasileira_2ed.pdf. Acesso em: 10 set. 2018.

BRASIL. Ministério do Desenvolvimento Social e Combate à Fome. Política nacional de assistência social PNAS/2004. Norma operacional básica NOB/SUAS. Brasília, DF, 2009. Disponível em: https://www.mds.gov.br/webarquivos/publicacao/assistencia social/ Normativas/PNAS2004.pdf.Acesso em: 13 out. 2018.

BUYUKTUNCER, Z.; AYAZ, A.; DEDEBAYRAKTAR, D.; NAN-EROGLU, E.; ELLAHI, B.; BESLER, B.T. Promoting a Healthy Diet in Young Adults: The Role of Nutrition Labelling. Nutrients, v. 10, n. 1335, p. 1-12, 2018.

CAMPBELL, K. J.; ABBOTT, G.; SPENCE, A. C.; CRAWFORD, D. A.; Crawford,MCNAUGHTON, S. A.; BALL, K. Home food availability mediates associations between mothers' nutrition knowledge and child diet. Appetite, v. 71, n. 1, p. 1-6, 2013.

CONSELHO NACIONAL DE SAÚDE (CNS). Resolução $\mathbf{n}^{\circ}$ 466, de 12 de dezembro de 2012. Disponível em: http://bvsms.saude.gov.br/bvs/saudelegis/cns/ 2013/res046612 12 2012.html. Acesso em: 15 nov. 2018.

KAC, G.; SICHIERI, R.; GIGANTE, D. P. Epidemiologia nutricional. Rio de Janeiro: Fiocruz; Atheneu, 2007.

LENG , G.; ADAN, R. A. H.; BELOT, M.; BRUNSTROM, J. M.; GRAAF, K.; DICKSON, S. L.; HARE T.; MENZIES, J.; PREISSL, H.; REISCH, L. A.; ROGERS, P. J.; SMEETS, P. A. M. The determinants of food choice. Proceedings of the Nutrition Societyv. 76, n. 3, p. 316-327, 2017.

LOUZADA, M. L. C.; BARALDI, L. G.; STEELE, E. M.; MARTINS, A. P.; CANELLA, D. S.; MOUBARAC, J. C.; LEVY, R. B.; CANNON, G.; AFSHIN, A.; IMAMURA, F.; 
Relação entre conhecimento materno sobre rotulagem de alimentos e consumo de ultraprocessados em crianças e adolescentes atendidos em um projetos de extensão universitária

MOZAFFARIAN, D.; MONTEIRO, C.A. Consumption of ultra-processed foods and obesity in Brazilian adolescents and adults. Preventive Medicine, v. 81, p. 9-15, 2015.

LOVELACE, S.; RABIEE-KHAN, F. Food choices made by low-income households when feeding their pre-school children: a qualitative study. Matern Child Nutr., v. 11, n. 4, p.870-881, 2015.

MARZAROTTOO, B.; ALVES, M. K. Leitura de rótulos de alimentos por frequentadores de um estabelecimento comercial. Ciência e Saúde, Porto Alegre, v. 10, n. 2, p. 102-108, 2017. Disponível

em: http://revistaseletronicas.pucrs.br/ojs/index.php/faenfi/article/view/24220/15600. Acesso em 28 out. 2019.

MILLER, L. M. S.; CASSADY, D. L. The effects of nutrition knowledge on food label use. A review of the literature.Appetite, v. 92, p. 207-216, 2015a. Disponível em: https://www.ncbi.nlm.nih.gov/pmc/articles/PMC4499482/pdf/nihms699704.pdf. Acesso em: 15 maio 2019.

MILLER, L.M.; CASSADY, D. L.; APPLEGATE, E. A.; BECKETT, E. A.; WILSON, M. D.; GIBSON, T. N.; ELLWOOD, K.Relationships among food label use, motivation, and dietary quality. Nutrients, v. 7, n. 2, p. 1068-1080, 2015b.

MONTEIRO, C.A.; CANNON, G.; LEVY, R.B.; CLARO, R.; MOUBARAC, J.C. The food system. Ultra-processing. The big issue for nutrition, disease, health, well-being. World Nutr,v.3, n.12, p.527-569, 2012.

NASCIMENTO, C.; RAUPP, S. M. M.; TOWNSEND, R. T.; BALSAN, G. A.; MINOSSI, V. Conhecimento de consumidores idosos sobre rotulagem de alimentos. Revista de Epidemiologia e Controle de Infecção, Santa Cruz do Sul, v. 3, n. 4, p. 144-147, 2013. Disponível em: http://www.ppgcardiologia.com.br/wp-content/uploads/2014/05/Conhecimento-de-consumidoresidosos-sobre-rotulagem-de-alimentos.pdf. Acesso em: 28 out. 2019.

NCD RISK FACTOR COLLABORATION. Trends in adult body-mass index in 200 countries from 1975 to 2014: a pooled analysis of 1698 population-based measurement studies with 19.2 million participants. Lancet, London, v. 387, p.1377-1396, 2016. Disponível em: https://www.thelancet.com/action/showPdf?pii=S0 140-6736\%2816\%2930054-X. Acesso em: 25 abr. 2019.

PHILIPPI, S. T. Tabela de composição de alimentos: suporte para decisão nutricional. 6. ed. São Paulo: Manole, 2018.

PINHEIRO, A. B. V.; LACERDA, E. M. A.; BENZECRY, E. H.; GOMES M. C. S.; COSTA, V. M.. Tabela para avaliação de consumo alimentar em medidas caseiras. 5. ed. Rio de Janeiro: Atheneu, 2005.

PINTO, S. P.; SILVA, R. C. R.; PRIORE, S. E.; ASSIS, A. M. O.; PINTO, E. J. Prevalência de pré-hipertensão e de hipertensão arterial e avaliação de fatores associados em crianças e adolescentes de escolas públicas de Salvador, Bahia, Brasil. Caderno de Saúde Pública, Rio de Janeiro, v. 27, n. 6, 2011. Disponível em: http://www.scielo.br/pdf/csp/v27n6/ 04.pdf. Acesso em: 25 abr. 2019. 
Relação entre conhecimento materno sobre rotulagem de alimentos e consumo de ultraprocessados em crianças e adolescentes atendidos em um projetos de extensão universitária

RAUBER, F.; CAMPAGNOLO, P.D.; HOFFMANN, D.J.; VITOLO, M. R.. Consumption of ultra-processed food products and its effects on children's lipid profiles: a longitudinal study. Nutrition, Metabolism \& Cardiovascular Diseases, n. 25, n. 1, p. 116-122, 2014.

RUSSELL, C.G.; WORSLEY, A.; LIEM, D.G. Parents' food choice motives and their associations with children's food preferences. Public Health Nutr., v. 18, n. 6, p. 10181027, 2015.

SPARRENBERGER, K. FRIEDRICH, R. R.; SCHIFFNER, M. D.; SCHUCH, I.; WAGNER, M. B. Ultra-processed food consumption in children from a Basic Health Unit. Jornal de Pediatria, [S.1.], v. 91, n. 6, 2015. Disponível em: http://www.scielo.br/pdf/jped/v91n6/00217557-jped-91-06-0535.pdf. Acesso em: 25 abr. 2019.

TAVARES, L. F.; FONSECA, S. C.; GARCIA ROSA, M. L.; YOKOO, E. M. Relationship between ultra-processed foods and metabolic syndrome in adolescents from a brazilian family doctor program. Public Health Nutrition, Cambridge, v. 15, n. 1, p. 82-87, 2011. Disponível em: $\quad$ https://www. cambridge.org/core/services/aop-cambridgecore/content/view/198EA399730DBF2C5488F0F1360C9B6D/S136898001

1001571a.pdf/relationship between ultraprocessed foods and metabolic syndrome in adoles cents from a brazilian family doctor program.pdf. Acesso em: 25 abr. 2019.

UNIVERSIDADE ESTADUAL DE CAMPINAS - UNICAMP. Tabela brasileira de composição de alimentos - TACO. 4. ed. rev. e ampl. Campinas: UNICAMP/NEPA, 2011. 161 p. Disponível em: https://www.cfn.org.br/wpcontent/uploads/2017/03/taco 4 edicao ampliada e revisada.pdf. Acesso em: 19 de dezembro de 2019.

VILLA, J. K. D.; SILVA, A. R.; SANTOS, T. S. S.; RIBEIRO, A. Q.; PESSOA, M. C.; SANT'ANA, L. F. R.Padrões alimentares de crianças e determinantes socioeconômicos, comportamentais e maternos. Revista Paulista de Pediatria, São Paulo, v. 33, n. 3, p. 302-209, 2015. Disponível em: http://www.scielo.br/pdf/rpp/v33n3/0103-0582-rpp-33-03-0302.pdf . Acesso em: 25 set. 2018.

YEE, A.Z.; LWIN, M.O.; HO, S.S.The influence of parental practices on child promotive and preventive food consumption behaviors: a systematic review and meta-analysis.Int J Behav Nutr Phys Act., v. 14, n. 1, p. 47, 2017.

Recebido em: 06/01/2020

Aceito em: 26/11/2020 